\title{
Cyclosporin A effects during primary and secondary activation of human umbilical cord blood T lymphocytes
}

\author{
Suzanne Kadereit ${ }^{\mathrm{a}}$, Margaret M. Kozik ${ }^{\mathrm{a}}$, \\ Gwendolyn R. Junge ${ }^{a}$, Robin E. Miller ${ }^{b}$, Laura F. Slivka ${ }^{a}$, Linda S. Bos ${ }^{a}$, \\ Kathleen Daum-Woods ${ }^{\text {a }}$, R. Michael Sramkoskic, James W. Jacobberger ${ }^{\mathrm{c}}$, and Mary J. Laughlin ${ }^{\mathrm{a}}$ \\ ${ }^{a}$ Department of Medicine, Case Western Reserve University/University Hospitals, Cleveland, Ohio, USA; \\ ${ }^{\mathrm{b}}$ Department of Pediatrics, Case Western Reserve University/University Hospitals, Cleveland, Ohio, USA; \\ 'Ireland Cancer Center Flow Cytometry Core Facility, Case Western Reserve University/University Hospitals, Cleveland, Ohio, USA
}

\begin{abstract}
Objective. Cyclosporin A (CsA), effective in prophylaxis and treatment of graft-vs-host disease (GVHD) after human allogeneic transplantation, blunts $T$-cell responses by inhibiting nuclear factor of activated T cells-1 (NFAT1) activation. This laboratory has shown that NFAT1 protein expression is severely reduced in human $\mathrm{UCB}$ (umbilical cord blood) $\mathrm{T}$ cells. Since UCB is increasingly used as a hematopoietic stem cell source in allogeneic transplantation, it is important to determine whether CsA sensitivity in UCB differs from that of adult $\mathrm{T}$ cells.

Methods. Surface flow cytometric analysis, intracellular cytokine staining, flow cytometric analysis of cell death, and thymidine incorporation were used in this study to determine T-cell activation and effector functions during primary and secondary stimulation in the presence of CsA. Results. Although we observed differential CsA sensitivity of T-cell activation marker (CD69, CD45RO, CD25) upregulation comparing UCB and adult, we did not observe any significant difference in CsA sensitivity of T-cell effector functions. Importantly, we observed reduced IFN- $\gamma$ and TNF- $\alpha$ expression in UCB T cells both in primary and secondary stimulation, as well as increased rates of activation-induced cell death (AICD).

Conclusion. Thus, our studies do not support the previous hypothesis that reduced GVHD observed after UCB transplantation is attributable to increased CsA sensitivity of UCB T cells. Rather, reduced UCB T-cell cytokine production and increased AICD may be important cellular mechanisms underlying these favorable rates of GVHD in UCB transplant recipients.
\end{abstract}

The immunosuppressive effect of cyclosporin A (CsA) on human $\mathrm{T}$ cells is mediated by forming complexes with cyclophilins, abundant intracellular enzymes possessing peptidyl-prolyl isomerase activity, which function as protein chaperones within the cell. This complex can bind to the calcium-dependent serine/threonine phosphatase calcineurin, thus preventing it from dephosphorylating and activating its substrate, the transcription factor NFAT1 (nuclear factor of activated T cells-1) $[1,2]$. NFAT1 regulates the transcriptional activation of a large number of inducible genes en-

Offprint requests to: Mary J. Laughlin, M.D., Director, Allogeneic Transplant Program, Case Western Reserve University, University Hospitals Ireland Comprehensive Cancer Center, 11100 Euclid Avenue, Cleveland, OH 44106-5065; E-mail: mj113@po.cwru.edu coding cytokines and cell-surface receptors that are essential for effective T-cell responses $[3,4]$.

Allogeneic stem-cell transplant recipients receive CsA immunosuppressive therapy as GVHD (graft-vs-host disease) prophylaxis and treatment [5]. Although CsA is effective in reducing the incidence and severity of GVHD, paradoxical GVHD flare has been observed upon abrupt withdrawal of CsA treatment, possibly due to the fact that CsA inhibits T-cell cytokine production but not antigenspecific priming [6]. Moreover, administration of CsA can aggravate delayed type hypersensitivity (DTH) responses and certain autoimmune diseases $[7,8]$.

Umbilical cord blood (UCB) from related and unrelated donors has recently emerged as a novel source of blood stem cells for human allogeneic transplantation. Despite human leukocyte antigen (HLA) mismatching, the incidence 
and severity of acute and chronic GVHD in UCB recipients is low [9-11]. One possible basis for this observation is the finding that activated UCB T cells produce reduced proinflammatory cytokines, as well as reduced alloantigen-specific cytotoxicity and proliferation, when compared to adult T cells [12-16]. A second possible basis for reduced GVHD has been suggested by McDouall et al., who observed increased CsA sensitivity of PHA-induced upregulation of CD25, interleukin (IL)-2 production and proliferation in UCB T cells. Since UCB transplant patients receive CsA for GVHD prophylaxis, increased CsA sensitivity of UCB T cells may contribute to the low incidence and severity of GVHD observed [17].

This laboratory recently reported severely reduced to absent NFAT1 protein expression in unstimulated UCB T cells, with significantly reduced upregulation upon primary stimulation, when compared to adult T cells [14]. It is therefore possible that in the absence of normal NFAT1 protein expression, the immunosuppressive effects of CsA may be altered in UCB T cells. It is thus important to further characterize UCB T-cell sensitivity to CsA, as UCB is increasingly used as a stem cell source for human allogeneic transplantation.

Therefore, we measured UCB T-cell activation marker upregulation and effector functions and their sensitivity to CsA inhibition, and compared this to adult T cells. Also, since unprimed UCB, compared to adult T-cell populations, lack significant numbers of memory $\mathrm{CD}^{2} 4 \mathrm{RO}^{+} \mathrm{T}$ cells [18], we measured secondary UCB T-cell responses, hypothesizing that after priming UCB would contain a larger proportion of T cells with memory phenotype. Interestingly, we did not observe a significant difference in CsA sensitivity of effector functions such as proliferation and activation-induced cell death (AICD) comparing UCB and adult T cells. Furthermore, cytokine production was in both cases inhibited to basal levels. However, we found that upon restimulation UCB T cells expressed reduced amounts of tumor necrosis factor- $\alpha$ (TNF- $\alpha$ ) and interferon- $\gamma$ (IFN- $\gamma$ ), while exhibiting stronger AICD, when compared to adult $\mathrm{T}$ cells. Our observations suggest that reduced GVHD after UCB transplantation is not attributable to an overall increased sensitivity of UCB T cells to CsA, but rather point to complex differences in gene regulation between UCB and adult $\mathrm{T}$ cells that may underlie the low incidence and severity of GVHD observed after UCB transplantation.

\section{Materials and methods}

\section{Cells and reagents}

Human umbilical cord blood and adult peripheral blood from healthy donors were collected according to institutional guidelines after informed consent. Mononuclear cells (MNC) were separated and used immediately, without freezing, as previously described [14]. Concanavalin A (ConA) (Sigma Chemical Co., St. Louis, MO, USA) titration (1-5 $\mu \mathrm{g} / \mathrm{mL})$ was performed to establish an optimal concentration of $2 \mu \mathrm{g} / \mathrm{mL}$, at which the cells proliferate robustly while maintaining viability, as assayed by thymidine incorporation and trypan blue exclusion. Cyclosporin A (CsA, Sigma) was used at the manufacturer's recommended dose of 1 $\mu \mathrm{g} / \mathrm{mL}$, at which we observed a complete inhibition of proliferative response up to $5 \mu \mathrm{g} / \mathrm{mL}$ of ConA. All stimulations were performed at $2 \mu \mathrm{g} / \mathrm{mL}$ of ConA in the presence of $1 \mu \mathrm{g} / \mathrm{mL}$ of CsA, where indicated.

\section{Total CD69 staining}

Resting T cells express intracellular stores of CD69, which upon stimulation are transported rapidly to the cell surface [19]. This suggests that additional regulation of CD69 expression occurs at the posttranscriptional level. We therefore measured total CD69 expression after permeabilization of the cells, as described [14], thus capturing surface and intracellular CD69 expression.

\section{Surface staining}

MNC were stimulated with ConA in bulk culture, at a concentration of $2 \times 10^{6}$ cells $/ \mathrm{mL}$ in complete RPMI (Gibco BRL, Gaithersburg, MD, USA) containing 10\% fetal bovine serum (Gibco BRL), $1 \mathrm{mM}$ sodium pyruvate (Gibco BRL), $0.1 \mathrm{mM}$ nonessential amino acids (Gibco BRL), $10 \mathrm{mM}$ HEPES (Gibco BRL), and $58 \mu \mathrm{M}$ 2-mercaptoethanol (Sigma), for the indicated time periods, in presence or absence of CsA. At the indicated time points, $1 \times 10^{6}$ $\mathrm{MNC}$ were stained with monoclonal antibody (mAb) against CD3APC, CD25-PE, and CD45RO-FITC (Pharmingen, San Diego, CA, USA) and 15,000 gated CD3 + events were acquired on an Elite ESP flow cytometer (Beckman Coulter, Miami, FL, USA). Data were analyzed with WinList (Verity Software House Inc., Topsham, ME, USA) and expressed as percent of gated CD3 ${ }^{+} \mathrm{T}$ cells. Inhibition by CsA was calculated as percent reduction of total positive staining after stimulation with ConA.

\section{Intracellular cytokine staining}

During primary or secondary stimulation $5 \mu \mathrm{g} / \mathrm{mL}$ of Brefeldin A (BFA, Sigma) was added for the final 16 hours of stimulation. Cells were washed, permeabilized, and stained as previously described [14] with anti-CD3-APC, anti-CD69-PE (Pharmingen), anti-IFN- $\gamma$-FITC, or anti-TNF- $\alpha$-FITC (R \& D Systems, Minneapolis, MN, USA). 15,000 gated CD3 ${ }^{+}$events were acquired on an Elite ESP flow cytometer and data were analyzed with WinList. Cells expressing positive cytoplasmic cytokine staining were calculated as percentage of gated $\mathrm{CD} 3^{+}$cells.

\section{T-cell proliferative response to ConA}

$\mathrm{MNC}$ were stimulated in triplicate at $0.2 \times 10^{6}$ cells/round-bottom well in $200 \mu \mathrm{L}$ complete RPMI medium with ConA in absence or presence of CsA for up to 96 hours. Cells were pulsed with $1 \mu \mathrm{Ci}$ / $\mathrm{mL}$ of ${ }^{3} \mathrm{H}$-thymidine (Amersham, Buckinghamshire, UK) for the final 24 hours of stimulation, harvested onto fiber mats, and ${ }^{3} \mathrm{H}$ thymidine incorporation measured.

\section{Seconday T-cell stimulation and AICD}

MNC were stimulated as described above, in absence of CsA. Secondary stimulation was carried out as described previously, with modifications [20]. Briefly, after 48 hours the cells were washed in $10 \mathrm{mg} / \mathrm{mL}$ of $\alpha$-methylmannoside and resuspended at $2 \times 10^{6}$ cells/mL in complete RPMI with $50 \mathrm{U} / \mathrm{mL}$ of IL-2 (Chiron, Emeryville, CA, USA). After 72 hours of IL-2 treatment, T-cell percentage increased to greater than $80 \%$ of viable lymphocytes, as 


\section{A}
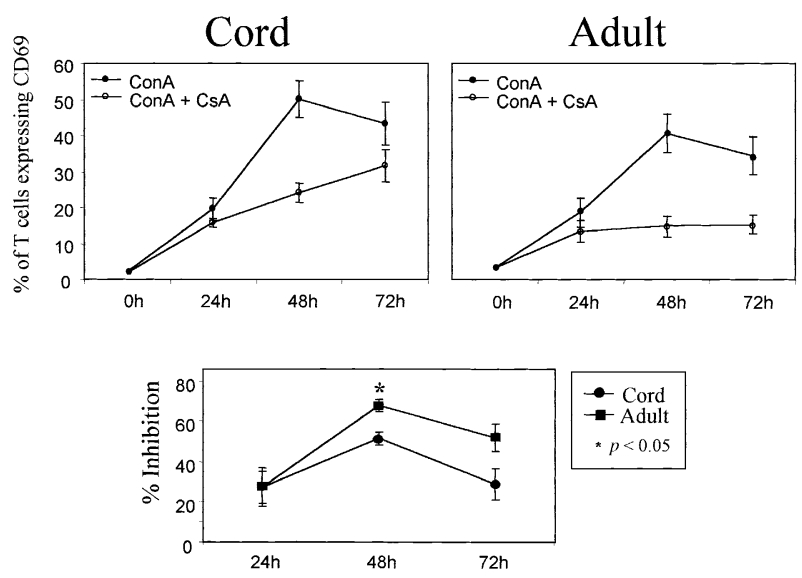

B
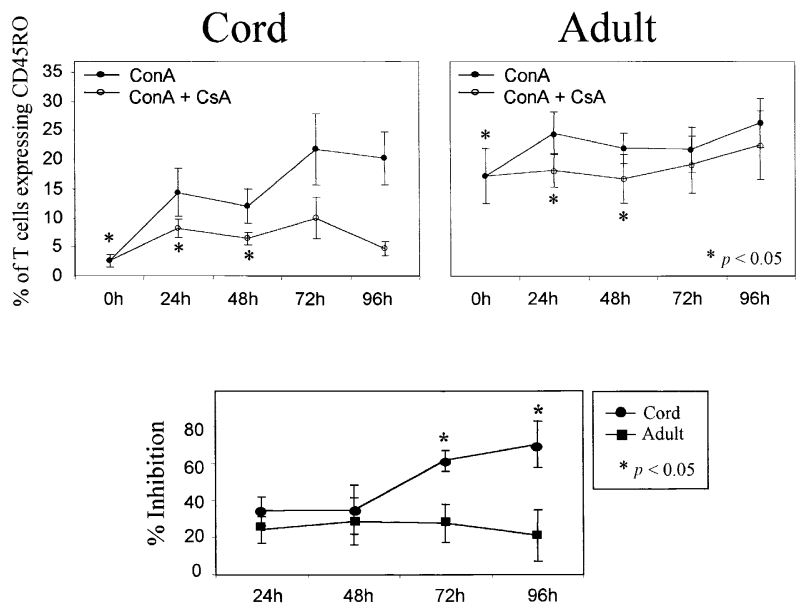

C
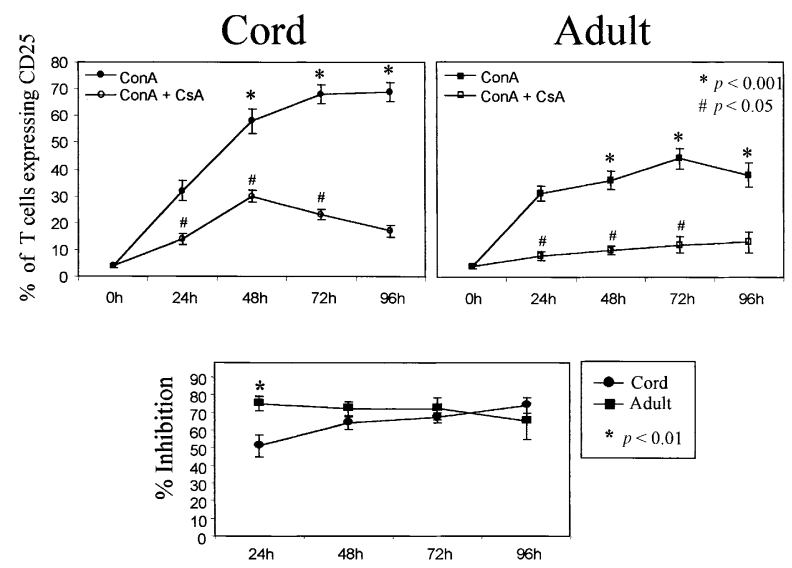

Figure 1. Differential CsA sensitivity of CD69, CD45RO, and CD25 upregulation in UCB T cells during primary stimulation. MNC from cord and adult were stimulated for 96 hours with ConA in presence or absence of CsA. Total CD69 expression in permeabilized gated CD3 ${ }^{+}$cells, as well as surface expression of CD45RO and CD25 on gated CD3 ${ }^{+}$cells, were measured by flow cytometry. CsA inhibition was calculated as percent inhibition of expression in absence of CsA. Data are represented as mean \pm SEM; asterisks indicate significant difference between UCB and adult. (A) Expression and inhibition of CD69 expression by CsA (cord $n=9$; adult $n=6$ ). (B) Expression and inhibition of CD45RO expression by CsA $(\mathrm{n}=7)$. (C) Expression an $\mathrm{d}$ inhibition of CD25 expression by CsA ( $\operatorname{cord} \mathrm{n}=12$; adult $\mathrm{n}=8$ ). assessed by flow cytometry (data not shown). Cells were washed and restimulated in complete RPMI with $2 \mu \mathrm{g} / \mathrm{mL}$ of ConA at a cell concentration of $2 \times 10^{6}$ cells $/ \mathrm{mL}$, in presence or absence of 1 $\mu \mathrm{g} / \mathrm{mL}$ of CsA. For quantification of AICD, $1 \times 10^{6}$ cells were stained with $5 \mu \mathrm{g} / \mathrm{mL}$ of propidium iodide and percent of apoptotic cells was acquired immediately on an XL-MCL flow cytometer (Beckman Coulter).

Statistical analyses

All experiments were repeated a minimum of three times, each with two different adult controls and UCB units. Values are the mean \pm SEM. Statistical significance was determined between the indicated values by two-tailed, nonpaired, unequal variance Student's $t$-test.

\section{Results}

Differential CsA sensitivity of UCB T-cell activation marker upregulation during primary stimulation Upregulation of the activation markers CD69, CD45RO, and CD25 was measured in gated CD3 + cells by flow cytometry after stimulation with ConA in the presence of CsA (see Materials and Methods). As previously described [21], we found equivalent percent of CD69 expressing T cells in cord and adult. Interestingly, we found a significantly reduced CsA sensitivity of CD69 upregulation in UCB when compared to adult $\mathrm{T}$ cells (Fig. 1A). In contrast, while CsA sensitivity of CD45RO upregulation was equivalent in UCB and adult during the first 48 hours of stimulation, there was significantly higher CsA sensitivity in UCB T cells at 72 and 96 hours of stimulation (Fig. 1B), when CD45RO expression on UCB T cells became equivalent to adult expression. Furthermore, we found decreased CsA sensitivity of CD25 upregulation in UCB T cells at 24 hours, but equivalent CsA sensitivity comparing cord and adult at time points 48 hours, 72 hours, and 96 hours of primary stimulation (Fig. 1C).

\section{Equivalent CsA sensitivity of proliferation} and cytokine production after primary stimulation In accordance with previous reports $[17,18]$, we measured equivalent percentages of CD25 expressing T cells in UCB and adult after 24 hours of stimulation. However, significantly higher percentages of $\mathrm{T}$ cells expressing CD25 were observed in UCB after 48 hours and 72 hours of primary stimulation (Fig. 1C). CD25 is the high-affinity chain of the T-cell growth factor IL-2. Accordingly, at these same time points, increased proliferation, as assessed by ${ }^{3} \mathrm{H}$-thymidine incorporation, was observed upon stimulation with ConA, when compared to adult. Despite equivalent percentages of $\mathrm{CD}^{+}$lymphocytes in UCB and adult at onset of proliferation, proliferation was found to be more than twofold stronger in UCB than in adult (Fig. 2). Moreover, in both UCB and adult, proliferation was effectively suppressed in the presence of CsA. 


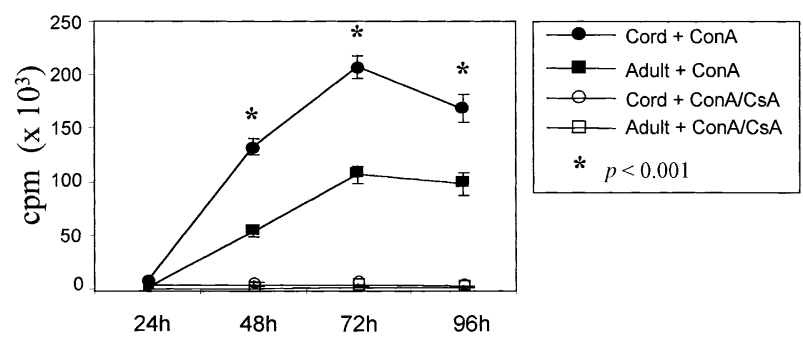

Figure 2. Increased proliferation in UCB during primary stimulation. MNC from cord and adult were stimulated for 96 hours as described, comparing at least $1 \mathrm{UCB}$ and 1 adult in each experiment. Proliferation was measured by ${ }^{3} \mathrm{H}$-thymidine incorporation. Asterisks indicate significant difference between UCB and adult (mean values from 4 individual experiments \pm SEM; $n=6$ ).

Production of TNF- $\alpha$ and IFN- $\gamma$, both pivotal cytokines in the pathogenesis of GVHD, can be successfully inhibited in adult peripheral $\mathrm{T}$ cells by $\mathrm{CsA}$ in vitro and in vivo [22]. However, in UCB T cells it is not clear to what extent TNF$\alpha$ and IFN- $\gamma$ are inhibited at the single-cell level by CsA. We therefore analyzed expression of cytoplasmic TNF- $\quad \alpha$ and IFN- $\gamma$ in $\mathrm{CD}^{+} \mathrm{T}$ cells. Although CD69 expression was equivalent, reduced percentages of cytoplasmic TNF- $\quad \alpha$ (Fig. 3) and IFN- $\gamma$ (data not shown) expressing T cells in stimulated UCB were observed up to 72 hours of primary stimulation, indicating that percentages of cytokine-producing T cells in UCB are reduced, rather than delayed. Furthermore, in the presence of CsA, TNF- $\alpha$ and IFN- $\gamma$ expression were reduced to basal levels in both UCB and adult T cells.

\section{Differential activation and effector}

functions during secondary stimulation, but equivalent CsA sensitivity between UCB and adult T cells MNC were analyzed by flow cytometry for CD69 and CD45RO expression after secondary stimulation (Materials and Methods). We observed only a slight difference in CD69 expression in UCB T cells after 24 hours of restimulation, $23 \%$ ( $\pm 2.2 \%$ ) of $\mathrm{T}$ cells expressing CD69 in UCB, compared to $32 \%$ ( $\pm 2.5 \%$ ) of CD69 expressing T cells in adult $(p=0.036)$. Furthermore, although percentages of CD45RO ${ }^{+}$T cells were found equivalent in UCB and adult after priming, significantly reduced further upregulation of CD45RO after 24 hours of secondary stimulation was noted on UCB T cells, when compared with adult, $22 \% \pm 1.9 \%$ vs $37 \% \pm 3.1 \%$, respectively ( $p=0.0028$ ). Upregulation of both activation markers was inhibited to the same extent by CsA in UCB and adult T cells (data not shown).
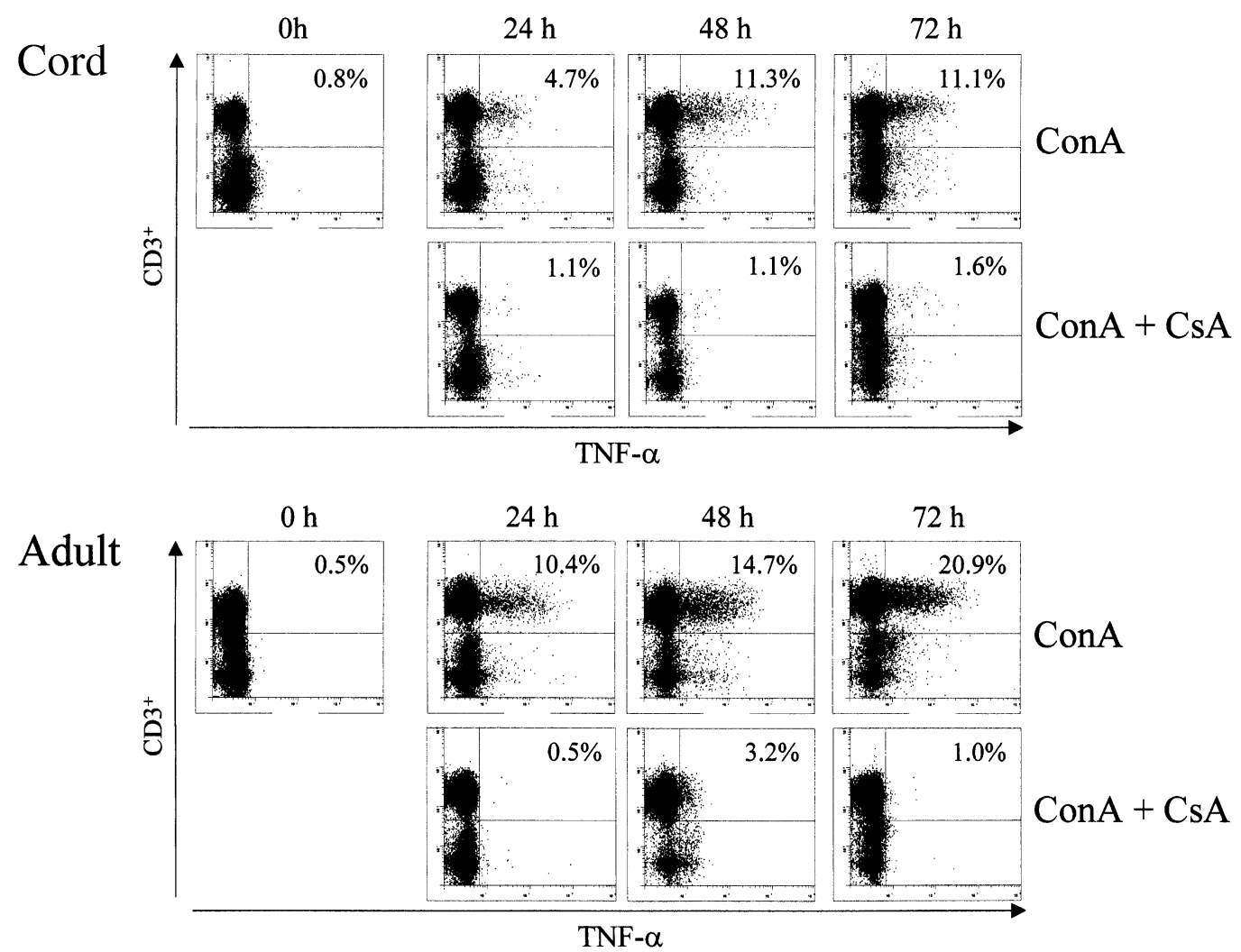

Figure 3. Equivalent CsA sensitivity of TNF- $\alpha$ expression between UCB and adult T cells during primary stimulation. Expression of intracellular cytokines in UCB and adult T cells during 72 hours of stimulation in presence or absence of CsA was measured by flow cytometry. Numbers $r$ epresent percentages of $\mathrm{CD}^{+}$cells positive for cytokine staining. Representative of 4 individual experiments, comparing each $2 \mathrm{UCB}$ and adult samples. 


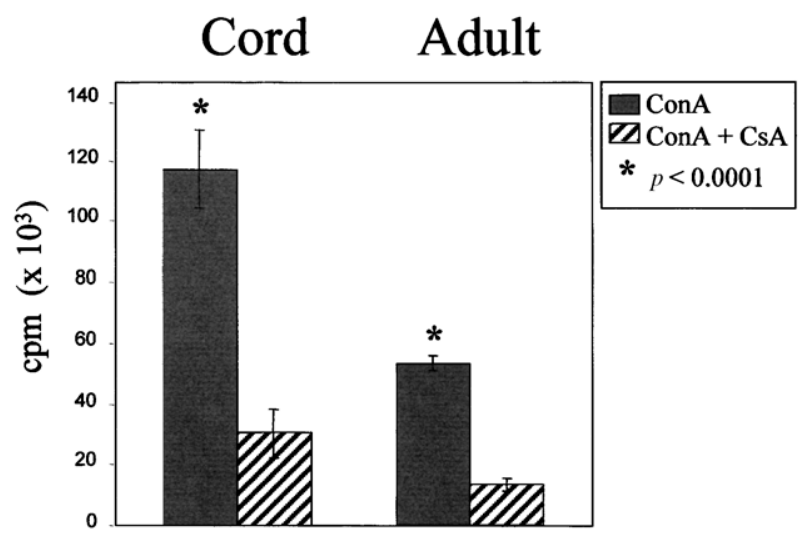

Figure 4. Increased proliferation in UCB T cells after secondary stimulation, but equivalent sensitivity to CsA. MNC from $2 \mathrm{UCB}$ and 2 adults were restimulated with ConA in presence or absence of CsA and proliferation assessed 24 hours after secondary stimulation by thymidine incorporation. Summarizes 4 individual experiments (mean values \pm SEM; $\operatorname{cord} n=$ 8 , adult $\mathrm{n}=8$ ). Asterisks indicate significant differences between cord and adult.

Next, proliferation was measured after restimulation. In contrast to a previous report describing reduced proliferation upon allogeneic restimulation [16], we measured a twofold greater proliferative response after 24 hours of mitogenic restimulation in UCB T cells compared with that in adult controls (Fig. 4). After that time point, proliferation decreased steadily in the cord blood and became equivalent to adult, which peaked only at 48 hours (data not shown). Interestingly, after primary stimulation and IL-2 culture, percentages of CD3 ${ }^{+}$were found equivalent in $\mathrm{UCB}$ and adult and stayed equivalent through secondary stimulation (data not shown). Again, the percentage of inhibition of proliferation by CsA was found equivalent between UCB and adult, $78 \%( \pm 4.76)$ and $73 \%( \pm 4.59)$ respectively.

Since unstimulated UCB T-cell populations lack significant expression of the memory phenotype marker CD45RO, suggestive of naivete, when compared to adult, we next analyzed cytokine expression in UCB T cells after priming and restimulation. After primary T-cell stimulation and IL-2 culture, UCB MNC contained equivalent percentages of CD45RO expressing T cells, when compared to adult (data not shown), suggesting similar memory phenotype between UCB and adult T cells. However, concurring with reduced CD69 and CD45RO upregulation upon restimulation, we found reduced percentages of cytokine-producing UCB T cells when compared to adult (Fig. 5). While we found a higher percentage of TNF- $\alpha$ - and IFN- $\gamma$-producing cells in adult $\mathrm{T}$ cells after secondary than after primary stimulation, suggestive of an expansion of cytokine-producing cells during primary stimulation, we found no significant augmentation of TNF- $\alpha$ - and IFN- $\gamma$-producing T cells in UCB. Moreover, the difference in percentages of cytokine-producing $\mathrm{T}$ cells between cord and adult was more pro- nounced after secondary stimulation than after primary stimulation. The presence of CsA reduced IFN- $\gamma$ and TNF$\alpha$ production to nearly basal levels in both UCB and adult (Table 1).

Appropriate AICD is crucial for maintenance of T-cell homeostasis in the periphery [20,23]. While several reports describe increased apoptosis in cord blood T cells [24,25], it is not clear if the presence of CsA during restimulation may affect this process in UCB T cells. Significantly higher AICD was measured in UCB T cells, already at baseline after IL-2 treatment, as well as after 24 hours of restimulation (Fig. 6). Kinetics of AICD in UCB mirrored the kinetics observed in adult $\mathrm{T}$ cells. Interestingly, in spite of inhibiting upregulation of Fas and FasL both in UCB and adult during primary and secondary stimulation (data not shown), CsA did not affect AICD in either UCB or adult (data not shown).

\section{Discussion}

McDouall et al. previously reported an increased CsA sensitivity of PHA-stimulated UCB T cells, compared to adult T cells. The authors described an increased inhibitory effect of low-dose CsA on UCB T-cell proliferation, CD25 upregulation, and IL-2 production [17]. The authors hypothesized that their observations may underlie the clinical observations of reduced GVHD after transplantation of HLA-disparate UCB allogeneic hematopoietic stem cells.

In contrast to this report, our findings in UCB T cells of reduced to absent expression of NFAT1 protein [14], a well described downstream target of CsA, suggested that UCB T cells might demonstrate altered sensitivity to the inhibitory effects of CsA. Furthermore, the effects of CsA on secondary $\mathrm{T}$-cell responses had not been previously investigated in UCB. We therefore investigated several aspects of primary and secondary T-cell responses in UCB and their sensitivity to the effects of CsA.

In our studies we did not detect a globally decreased or increased sensitivity of UCB T cells to the inhibitory effects of CsA. While there was differential CsA sensitivity of activation marker upregulation comparing UCB and adult T cells, we did not observe a significant difference between $\mathrm{UCB}$ and adult $\mathrm{T}$ cells in the effects of CsA on effector functions, including proliferation and AICD. Moreover, although cytokine production was found strongly reduced in UCB T cells upon primary and secondary stimulation, it was further reduced to basal levels by CsA, resulting in similar percentages of positive staining cells as in CsA-inhibited adult $\mathrm{T}$ cells. In the case of IFN- $\gamma$ production, inhibition by CsA was less evident in UCB T cells, due to the already very low percentages of positive staining cells. Our results strongly suggest that reduced GVHD observed after UCB transplantation cannot be attributed to increased CsA sensitivity of UCB T cells.

We observed significantly higher rates of proliferation in 


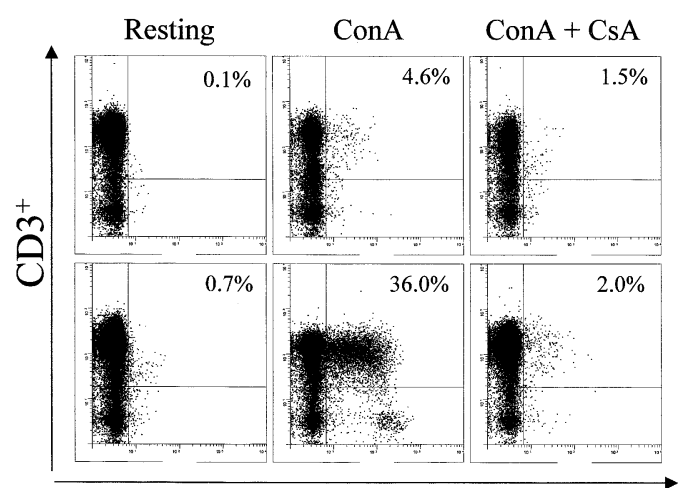

Cord

Adult

TNF- $\alpha$

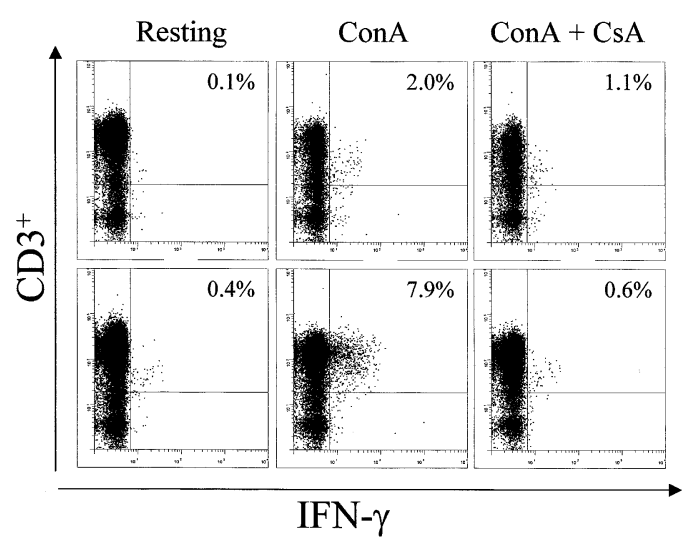

Cord

Adult

Figure 5. Reduced cytokine expression in UCB T cells after secondary stimulation. MNC from cord and adult were restimulated in presence or absence of CsA. Intracellular TNF- $\alpha$ and IFN- $\gamma$ expression were measured by flow cytometry after 24 hours. Percentages indicate calculated percentage of $\mathrm{CD} 3{ }^{+}$cells expressing cytokine. Representative of 3 experiments, comparing each 2 cords and 2 adults.

UCB after primary as well as secondary stimulation, associated with reduced percentages of effector cytokine-producing $\mathrm{T}$ cells. These observations are particularly relevant to recent reports demonstrating the association of effector cy-

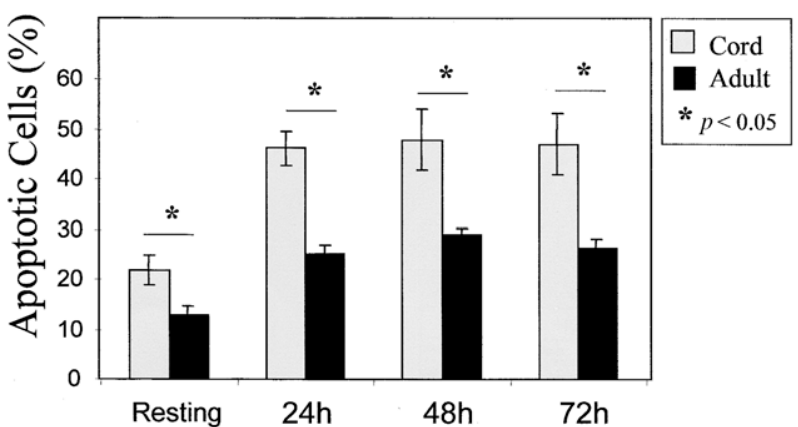

Figure 6. Increased AICD in UCB T cells after secondary stimulation. MNC were stimulated and rested as described in Materials and Methods and propidium iodide incorporation measured with flow cytometry, before restimulation (resting) and over 72 hours after restimulation with ConA (mean values $\pm \mathrm{SEM} ; \mathrm{n}=7$ ).

tokine expression with T-cell division [26-28]. Our results of reduced numbers of cytokine-expressing T cells in UCB after secondary stimulation suggest that, in contrast to adult $\mathrm{T}$ cells, the already reduced numbers of cytokine-producing UCB T cells observed after primary stimulation did not expand further, despite a strong overall proliferation during primary stimulation.

Furthermore, we observed no differential effect of CsA on the increased AICD we observed in UCB T cells. In contrast, CsA was noted to slightly increase AICD in both UCB and adult $\mathrm{T}$ cells. However, this trend of increased AICD in presence of CsA was not statistically significant. Further analyses of AICD in the presence of CsA are warranted, to verify if an increase in AICD in the presence of CsA may augment the immunosuppressive effects of CsA. Likewise, our observations of increased AICD in UCB T cells after secondary stimulation point to further possible underlying etiologies to the reduced GVHD observed after UCB transplantation, by rapid elimination of activated alloreactive donor $\mathrm{T}$ cells.

Our findings of increased cell division in concert with reduced cytokine production support the concept that the neo-

Table 1. Reduced cytoplasmic expression of IFN- $\gamma$ and TNF- $\alpha$ by UCB T cells after secondary stimulation

\begin{tabular}{|c|c|c|c|c|c|c|c|c|c|c|c|c|}
\hline & \multicolumn{12}{|c|}{ Activated T cells expressing cytoplasmic IFN- $\gamma(\%)$} \\
\hline & $\mathrm{CB} 1$ & $\mathrm{CB} 2$ & $\mathrm{AB} 1$ & $\mathrm{AB} 2$ & CB3 & CB4 & AB3 & AB4 & CB5 & CB6 & AB5 & AB6 \\
\hline Resting & 0.3 & 0.1 & 0.4 & 0.4 & 0.7 & 0.1 & 0.1 & 0.0 & 0.9 & 0.2 & 0.0 & 0.1 \\
\hline ConA & 3.2 & 2.0 & 7.9 & 9.9 & 0.9 & 1.7 & 3.6 & 2.2 & 4.1 & 1.2 & 4.4 & 3.9 \\
\hline \multirow[t]{3}{*}{ Con $\mathrm{A}+\mathrm{Cs} \mathrm{A}$} & 3.0 & 1.1 & 0.6 & 0.7 & 0.5 & 1.5 & 0.2 & 0.6 & 3.1 & $\mathrm{ND}$ & 0.7 & 2.2 \\
\hline & \multicolumn{12}{|c|}{ Activated T cells expressing cytoplasmic TNF- $\alpha(\%)$} \\
\hline & CB1 & $\mathrm{CB} 2$ & $\mathrm{AB} 1$ & $\mathrm{AB} 2$ & CB3 & CB4 & AB3 & $\mathrm{AB} 4$ & CB5 & CB6 & AB5 & AB6 \\
\hline Resting & 0.4 & 0.1 & 0.7 & 0.5 & 0.1 & 0.2 & 0.1 & 0.1 & 0.5 & 0.1 & 0.1 & 0.1 \\
\hline ConA & 6.7 & 4.6 & 36.0 & 32.5 & 0.7 & 2.3 & 17.8 & 10.4 & 3.1 & 1.8 & 12.7 & 7.3 \\
\hline ConA + CsA & 4.5 & 1.5 & 1.9 & 2.4 & 0.2 & 1.0 & 0.1 & 0.3 & 2.2 & 1.2 & 0.4 & 1.6 \\
\hline
\end{tabular}

Representation of three individual experiments each comparing 2 UBC (CB) and 2 adults $(\mathrm{AB})$. Percentages are of gated $\mathrm{CD}^{3+}$ lymphocytes $(\mathrm{ND}$, not determined). 
natal $\mathrm{T}$ cell may have a higher self-replicative capacity than its adult counterpart, in agreement with the necessity to populate the periphery of the neonate, while maintaining a window of tolerance against environmental antigens. In conjunction with this observation, we and others have described deficiencies in cellular signaling pathways including defective activation of Ras, PLC $\quad \gamma$, CD3 $\epsilon$, p56lck, ZAP-70, ERK2, SAPK/JNK, and p38 MAPK, as well as decreased Lck and NFAT1 protein expression [14,29-31]. These profound deficiencies in cellular signaling, in addition to the reduced effector functions described herein including proinflammatory cytokine production, combined with increased clearance of activated UCB T cells by AICD, in concert may underlie the reduced incidence and severity of GVHD observed after allogeneic transplantation of HLA-mismatched umbilical cord blood.

\section{Acknowledgments}

Supported by Grant No. 6230-98 from The Leukemia and Lymphoma Society and Grant RO1-AI47289-01 from the NIH/NIAID. M.J. Laughlin, M.D. is a Stephen Birnbaum Translational Research Investigator of the Leukemia and Lymphoma Society and Leukemia Scholar in Clinical Research.

The authors wish to thank the Labor and Delivery staff of University Hospitals of Cleveland/McDonald's Women and Children Hospital for their enthusiastic support.

\section{References}

1. Flanagan WM, Corthesy B, Bram RJ, Crabtree GR (1991) Nuclear association of a T-cell transcription factor blocked by FK-506 and cyclosporin A. Nature 352:803

2. Schreiber S, Crabtree G (1992) The mechanism of action of cyclosporin A and FK506. Immunol Today 13:136

3. Hodge MR, Ranger AM, de la Brousse FC, Hoey T, Grusby MJ, Glimcher LH (1996) Hyperproliferation and dysregulation of IL-4 expression in NF-ATP-deficient mice. Immunity 4:397

4. Rao A, Luo C, Hogan PG (1997) Transcription factors of the NFAT family: regulation and function. Ann Rev Immunol 15:707

5. Chao N, Snyder D, Jain M, et al. (2000) Equivalence of 2 effective graft-versus-host disease prophylaxis regimens: results of a prospective double-blind randomized trial. Biol Blood Marrow Transplant $6: 254$

6. Hess A, Jones RC, Santos G (1993) Autologous graft-vs-host disease: mechanisms and potential therapeutic effect. Bone Marrow Transplant 12 Suppl 3:S65

7. Prud'homme G, Parfrey N, Vanier L (1991) Cyclosporin-induced autoimmunity and immune hyperreactivity. Autoimmunity 9:345

8. Prud'homme G, Vanier L (1993) Cyclosporine, tolerance, and autoimmunity. Clin Immunol Immunopathol 66:185

9. Kurtzberg J, Laughlin M, Graham ML, et al. (1996) Placental blood as a source of hematopoietic stem cells for transplantation into unrelated recipients. N Engl J Med 335:157

10. Rubinstein P, Carrier C, Scaradavou A, et al. (1998) Outcomes among 562 recipients of placental-blood transplants from unrelated donors. $\mathrm{N}$ Engl J Med 339:1565

11. Rocha V, Wagner JJ, Sobocinski K, et al. (2000) Graft-versus-host dis- ease in children who have received a cord-blood or bone marrow transplant from an HLA-identical sibling. Eurocord and international bone marrow transplant registry working committee on alternative donor and stem cell sources. N Engl J Med 342:1846

12. Chalmers IMH, Janossy G, Contreras M, Navarrete C (1998) Intracellular cytokine profile of cord and adult blood lymphocytes. Blood 92:11

13. Ferrara JLM, Krenger W (1998) Graft-versus-host disease: the influence of type 1 and type $2 \mathrm{~T}$ cell cytokines. Transfusion Medicine Reviews 12:1

14. Kadereit S, Mohammad S, Miller R, et al. (1999) Reduced NFAT1 protein expression in human umbilical cord blood $\mathrm{T}$ lymphocytes. Blood 94:3101

15. Risdon G, Gaddy J, Stehman B, Broxmeyer HE (1994) Proliferative and cytotoxic responses of human cord blood T lymphocytes following allogeneic stimulation. Cell Immunol 154:14

16. Risdon G, Gaddy J, Horie M, Broxmeyer HE (1995) Alloantigen priming induces a state of unresponsiveness in human umbilical cord blood T cells. Proc Natl Acad Sci U S A 92:2413

17. McDouall R, Suitters A, Smith H, Yacoub M, Rose M (1994) Increased cyclosporine sensitivity of $\mathrm{T}$ cells from cord blood compared with those from adult. Clin Exp Immunol 95:519

18. Paiva A, Freitas A, Loureiro A, et al. (1998) Functional aspects of cord blood lymphocytes responses to polyclonal and allogeneic activation. Bone Marrow Transplant 22 Suppl 1:S31

19. Risso A, Smilovich D, Capra M, et al. (1991) CD69 in resting and activated T lymphocytes. Its association with a GTP binding protein and biochemical requirements for its expression. J Immunol 146:4105

20. Zheng L, Fisher G, Miller R, Peschon J, Lynch D, Lenardo M (1995) Induction of apoptosis in mature $\mathrm{T}$ cells by tumour necrosis factor. Nature 377:348

21. Cairo MS, Wagner JE (1997) Placental and/or umbilical cord blood: an alternative source of hematopoietic stem cells for transplantation. Blood 90:4665

22. Quesniaux V (1993) Immunosuppressants: tools to investigate the physiological role of cytokines. Bioessays 15:731

23. Ju S, Matsui K, Ozdemirli M (1999) Molecular and cellular mechanisms regulating $\mathrm{T}$ and $\mathrm{B}$ cell apoptosis trhough Fas/FasL interaction. Int Rev Immunol 18:485

24. Aggarwal S, Gupta A, Nagata S, Gupta S (1997) Programmed cell death (apoptosis) in cord blood lymphocytes. J Clin Immunol 17:63

25. Hagihara M, Chargui J, Gansuvd B, et al. (1999) Umbilical cord blood $\mathrm{T}$ lymphocytes are induced to apoptosis after being allo-primed in vitro. Bone Marrow Transplant 24:1229

26. Bird J, Brown D, Mullen A, et al. (1998) Helper T cell differentiation is controlled by the cell cycle. Immunity 9:229

27. Richter A, Lohning M, Radbruch A (1999) Instructions for cytokine expression in $\mathrm{T}$ helper lymphocytes in relation to proliferation and cell cycle progression. J Exp Med 190:1439

28. Cho B, Rao V, Ge Q, Eisen H, Chen J (2000) Homeostasis-stimulated proliferation drives naive $\mathrm{T}$ cells to differentiate directly into memory T cells. J Exp Med 192:543

29. Porcu P, Gaddy J, Broxmeyer HE (1998) Alloantigen-induced unresponsiveness in cord blood T lymphocytes is associated with defective activation of Ras. Proc Natl Acad Sci U S A 95:4538

30. Miscia S, Di Baldassare A, Sabatino G, et al. (1999) Inefficient phospholipase $\mathrm{C}$ activation and reduced LCK expression characterize the signaling defect of umbilical cord blood T lymphocytes. J Immunol $163: 2416$

31. Sato K, Nagayama H, Takahashi T (1999) Aberrant CD3- and CD28mediated signaling events in cord blood $\mathrm{T}$ cells are associated with dysfunctional regulation of Fas ligand mediated cytotoxicity. J Immunol 162:4464 